\title{
ON THE PERIODIC SOLUTIONS OF LINEAR HOMOGENEOUS SYSTEMS OF DIFFERENTIAL EQUATIONS
}

\author{
A.K. BOSE \\ Department of Mathematical Sciences \\ Clemson University \\ Clemson, South Carolina 29613 U.S.A. \\ (Received October 13, 1981)
}

ABSTRACT. Given a fundamental matrix $\phi(x)$ of an $n-t h$ order system of linear homogeneous differential equations $Y^{\prime}=A(x) Y$, a necessary and sufficient condition for the existence of a $k$-dimensional $(k \leq n)$ periodic sub-space (of period $T$ ) of the solution space of the above system is obtained in terms of the rank of the scalar matrix $\phi(T)-\phi(0)$.

KEY WORVS AND PHRASES. Linear homogeneous system of differential equations, Fundamental matrix, Periodic solutions, periodic sub-spaces of (period T), Rank of the scaiar matrix, Linearly independent vectors.

1980 MATHEMATICS SUBJECT CLASSIFICATION CODES. 34A20.

1. INTRODUCTION.

Consider the $n$-th order system of linear homogeneous differential equations

$$
\mathrm{Y}^{\prime}=\mathrm{A}(\mathrm{x}) \mathrm{Y} \text {, }
$$

where $Y=\operatorname{col}\left(y_{1}(x), y_{2}(x), \ldots, y_{n}(x)\right), Y^{\prime}=\operatorname{col}\left(Y_{1}^{\prime}(x), \ldots, y_{n}^{\prime}(x)\right), A(x)=\left(\left(a_{i j}(x)\right)\right)$ is a square matrix of order $n$, each element $a_{i j}(x)$ of $A(x)$ is a real-valued function continuous on the real line $R$. Let $S_{n}$ be the solution space of the system of equations (1.1) on the real line $\mathrm{R}$ and $\mathrm{T}>0$ be a real number. Let

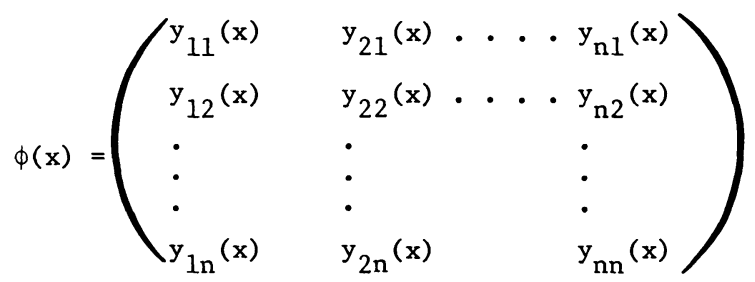


be a fundamental matrix of the system (1.1). The column vectors of $\phi(x)$ are 1 inearly independent solutions of (1.1).

The purpose of this note is to deduce a necessary and sufficient condition for the existence of periodic sub-spaces (of period $T$ ) of the solution space $S_{n}$ of the system (1.1) and to show that the existence and dimensions of these periodic sub-spaces depend not on any prior assumption about the periodicity (of period T) of the elements $a_{i j}(x)$ of the coefficient matrix $A(x)$ of the system (1.1) (that is, all the elements $a_{i j}(x)$ of $A(x)$ need not be periodic of period $T$ ), but precisely on the rank of the scalar matrix

$$
\phi(T)-\phi(0)
$$

\section{MAIN RESULTS.}

The condition (1.3) is stated more explicitly in the following theorem:

THEOREM. Let $\mathrm{k}$ be a non-negative integer, $0 \leq \mathrm{k} \leq \mathrm{n}$. There exists a $\mathrm{k}$-dimensional sub-space $S_{k}$ of the solution space $S_{n}$ of the linear homogeneous system (1.1) such that each member of $s_{k}$ is periodic of period $T$ and no member of $S_{n}-S_{k}$ is periodic of period $\mathrm{T}$ if and only if the rank of the scalar matrix $\phi(\mathrm{T})-\phi(0)$ is $\mathrm{n}-\mathrm{k}$.

The above theorem can also be phrased is terms of the eigen values of the scalar matrix $\phi^{-1}(0) \phi(T)$ as follows.

COROLLARY. Let $\mathrm{k}$ be a non-negative integer, $0 \leq \mathrm{k} \leq \mathrm{n}$. There exists a $\mathrm{k}$-dimensional sub-space $\mathrm{S}_{\mathrm{k}}$ of the solution space $\mathrm{S}_{\mathrm{n}}$ of the linear homogeneous system (1.1) such that each member of $S_{k}$ is periodic of period $T$ and no member of $S_{n}-S_{k}$ is periodic of period $\mathrm{T}$ if and only if $\lambda=1$ is an eigen value of the scalar matrix $\phi^{-1}(0) \phi(\mathrm{T})$ of multiplicity $\mathrm{k}$.

PROOF OF THE THEOREM. Let $\mathrm{k}$ be a non-negative integer, $0 \leq \mathrm{k} \leq \mathrm{n}$ and rank of $\phi(T)-\phi(0)$ is $n-k$. Then the dimension of the kernel of $\phi(T)-\phi(0)$ is $k$. Hence there exists $k$ linearly independent vectors

$$
v_{i}=\operatorname{col}\left(c_{i 1}, c_{i 2}, \ldots, c_{i n}\right), \quad i=1,2, \ldots, k,
$$

belonging to $\mathrm{R}^{\mathrm{n}}$ such that

$$
(\phi(T)-\phi(0)) v_{i}=0, \quad i=1,2, \ldots, k
$$


Let $f_{i}(x)=\phi(x) v_{i}, i=1,2, \ldots, k$. The linear independence of the vectors $v_{1}, v_{2}, \ldots, v_{k}$ implies the linear independence of the $k$ solution vectors $f_{1}(x), f_{2}(x), \ldots, f_{k}(x)$ of the system (1.1). Also,

$$
f_{i}(T)-f_{i}(0)=(\phi(T)-\phi(0)) v_{i}=0, \quad i=1,2, \ldots, k,
$$

implies by the uniqueness of the solutions of initial value problem that $f_{i}(x+T)-f_{i}(x)=0$ for all $x, i=1,2, \ldots, k$. Hence each solution vector $f_{i}(x)$, $i=1,2, \ldots, k$, is periodic of period $T$. Let $s_{k}$ be the $k$-dimensional periodic (of period $T$ ) sub-space of $S_{n}$ generated by $f_{1}(x), \ldots, f_{k}(x)$. We need to show that no member of $S_{n}-S_{k}$ is periodic of period T. Let $g_{1}(x) \varepsilon S_{n}-s_{k}$. Then $g_{1}(x)$ is nontrivial and $f_{1}(x), f_{2}(x), \ldots, f_{k}(x), g_{1}(x)$ are $k+1$ linearly independent members of $\mathrm{S}_{\mathrm{n}} \cdot$ Let

$$
g_{1}(x)=\phi(x) v_{k+1} \text {, where } v_{k+1}=\operatorname{col}\left(c_{k+1} c_{k+1} 2, \cdots, c_{k+1} n\right) .
$$

If possible, let $g_{1}(x)$ be periudic of period $T$. That is

$$
g_{1}(x+T)-g_{1}(x)=0 \text { for all } x .
$$

Then

$$
g_{1}(T)-g_{1}(0)=0 \text {. }
$$

Since any set of linearly independent members of $\mathrm{s}_{\mathrm{n}}$. form a part of a basis of $s_{n}$, let $f_{1}(x), f_{2}(x), \ldots, f_{k}(x), g_{1}(x), g_{2}(x), \ldots, g_{n-k}(x)$ be a basis of $s_{n}$ and

$$
g_{i}(x)=\phi(x) v_{k+i}, \quad i=2,3, \ldots, n-k,
$$

where $v_{k+i}=\operatorname{col}\left(c_{k+i} 1, c_{k+i} 2, \ldots, c_{k+i}\right), i=2,3, \ldots, n-k$. The linear independence of the basis vectors $f_{1}(x), f_{2}(x), \ldots, f_{k}(x), g_{1}(x), \ldots, g_{n-k}(x)$ implies that the matrix

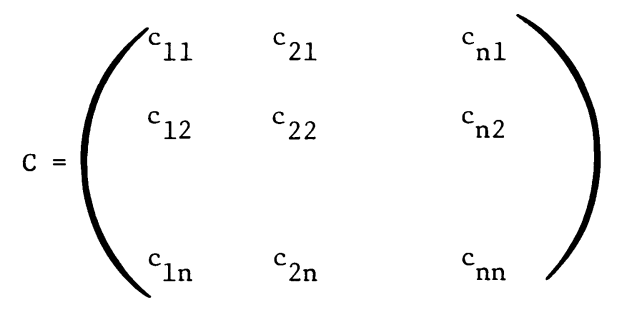

is non-singular and hence

$$
\text { rank of }(\phi(T)-\phi(0))=\text { rank of }(\phi(T)-\phi(0)) C, \quad[\text { see, } 2] \text {, }
$$


But, by actual multiplication and using (2.2) and (2.3), we see that the first $k+1$ column vectors of $(\phi(T)-\phi(0)) C$ are zero-vectors and hence the rank of $(\phi(T)-\phi(0)) C$ is at most $n-k-1$. Therefore, from (2.4)

$$
\mathrm{n}-\mathrm{k}=\text { rank of }(\phi(T)-\phi(0))=\text { rank of }(\phi(T)-\phi(0)) \mathrm{C} \leq \mathrm{n}-\mathrm{k}-1
$$

implying a contradiction. Hence $g_{1}(x)$ cannot be periodic of period $T$. That is no member of $S_{n}-S_{k}$ is periodic of period $T$.

Conversely, suppose that $S_{k}$ be a $k$-dimensional sub-space of $S_{n}$ such that every member of $S_{k}$ is periodic of period $T$ and no member of $S_{n}-S_{k}$ is periodic of period T. We need to show that the rank of $\phi(T)-\phi(0)$ is $n-k$.

Let $f_{1}(x), f_{2}(x), \ldots, f_{k}(x)$ be a basis of $s_{k}$ and $f_{1}(x), f_{2}(x), \ldots, f_{k}(x), g_{1}(x), \ldots$ $g_{n-k}(x)$ be a basis of $s_{n}$. Clearly $g_{i}(x) \varepsilon s_{n}-s_{k}, i=1,2, \ldots, n-k$. Hence each $g_{i}(x), i=1,2, \ldots, n-k$, is not periodic of period $T$. Again the $n-k$ vectors

$$
g_{1}(T)-g_{1}(0), g_{2}(T)-g_{2}(0), \ldots, g_{n-k}(T)-g_{n-k}(0)
$$

are linearly independent. For

$$
\ell_{1}\left(g_{1}(T)-g_{1}(0)\right)+\ell_{2}\left(g_{2}(T)-g_{2}(0)\right)+\ldots+\ell_{n-k}\left(g_{n-k}(T)-g_{n-k}(0)\right)=0
$$

implies by the uniqueness of the solutions of initial value problem that

$$
g(x)=l_{1} g_{1}(x)+\ldots+\ell_{n-k} g_{n-k}(x)
$$

is a periodic solution of the system (1.1) of period $\mathrm{T}$. Hence by our hypothesis $g(x) \varepsilon S_{k}$ and therefore

$$
g(x)=l_{1} g_{1}(x)+\ldots .+l_{n-k} g_{n-k}=b_{1} f_{1}(x)+\ldots+b_{k} f_{k}(x),
$$

for all $\mathrm{x}$, where $\mathrm{b}_{1}, \mathrm{~b}_{2}, \ldots, \mathrm{b}_{\mathrm{k}}$ are real constants.

Since $f_{1}(x), \ldots, f_{k}(x), g_{1}(x), \ldots, g_{n-k}(x)$ form a basis of $s_{n}$, it follows that

$$
\begin{aligned}
& \ell_{i}=0, \quad i=1,2, \ldots, n-k \\
& b_{j}=0, \quad j-1,2, \ldots, k .
\end{aligned}
$$

Hence the $n-k$ vectors

$$
g_{1}(T)-g_{1}(0), g_{2}(T)-g_{2}(0), \ldots, g_{n-k}(T)-g_{n-k}(0)
$$

are linearly independent.

Let $H(x)$ be the fundamental matrix of the linear system (1.1) whose column vectors are 


$$
f_{1}(x), f_{2}(x), \ldots, f_{k}(x), g_{1}(x), \ldots, g_{n-k}(x)
$$

and $\mathrm{C}$ be a non-singular scalar matrix such that

$$
H(x)=\phi(x) C .
$$

Then

$$
H(T)-H(0)=(\phi(T)-\phi(0)) C
$$

Since C is non-singular,

rank of $(\phi(T)-\phi(0))=$ rank of $(\phi(T)-\phi(0)) C=\operatorname{rank}$ of $(H(T)-H(0))$.

But, the first $k$ columns of $H(T)-H(0)$ are zero vectors by the periodicity of $f_{1}(x), f_{2}(x), \ldots, f_{k}(x)$ and the last $n-k$ column vectors

$$
g_{1}(T)-g_{1}(0), g_{2}(T)-g_{2}(0), \ldots, g_{n-k}(T)-g_{n-k}(0)
$$

of $\mathrm{H}(\mathrm{T})-\mathrm{H}(0)$ are linearly independent as proved before. Hence the rank of $H(T)-H(0)$ is $n-k$. That is, the rank of $\phi(T)-\phi(0)$ is $n-k$. This completes the proof of the theorem.

To prove the corollary, we see, from (2.1) that

$$
\phi^{-1}(0) \phi(T) v_{i}=v_{i}, \quad i=1,2, \ldots, k \text {. }
$$

That is,

$$
\left(\phi^{-1}(0) \phi(T)-I\right) v_{i}=0, \quad i=1,2, \ldots, k \text {. }
$$

where $I$ is the identity matrix. This means that $\lambda=1$ must be an eigen value of the scalar matrix $\phi^{-1}(0) \phi(T)$ of multiplicity $k$. Hence arguing similarly as in the proof of the theorem one can prove the corollary easily.

\section{REFERENCES}

1. CODDINGTON, EARL A. and LEVINSON, NORMAN. Theory of Ordinary Differential Equations, McGraw-Hil1 Book Company, New York.

2. BIRKHOFF, GARRETT and MACLANE, SAUNDERS. A Survey of Modern Algebra, Macmillan Company, New York. 


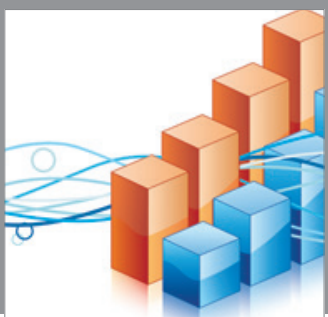

Advances in

Operations Research

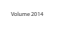

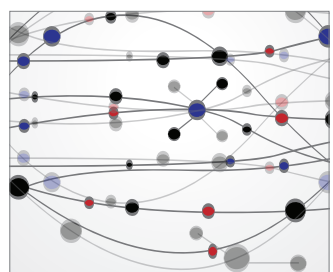

\section{The Scientific} World Journal
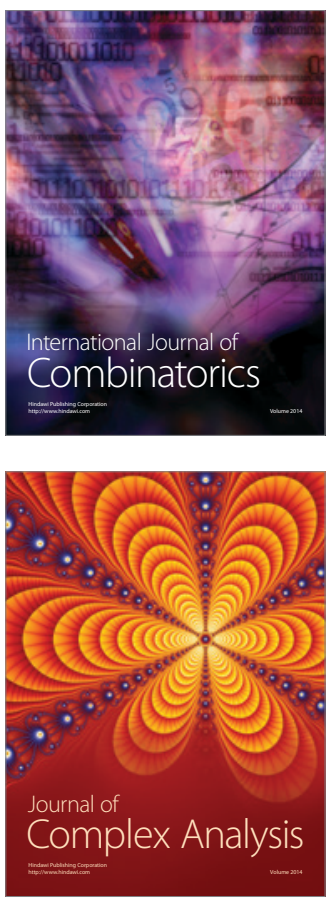

International Journal of

Mathematics and

Mathematical

Sciences
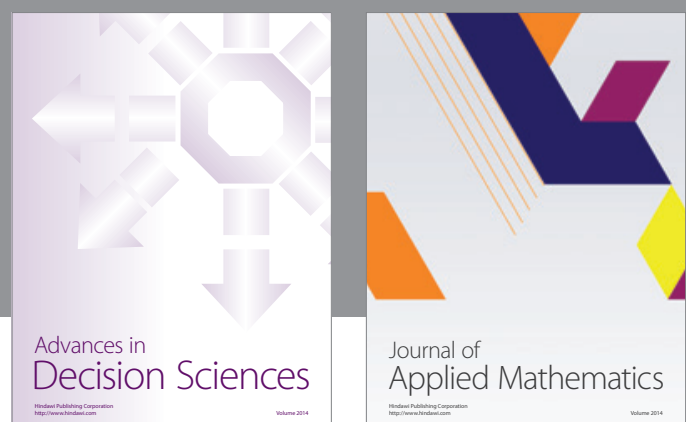

Journal of

Applied Mathematics
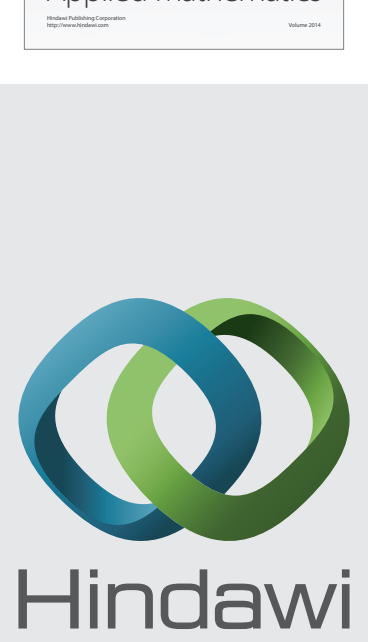

Submit your manuscripts at http://www.hindawi.com
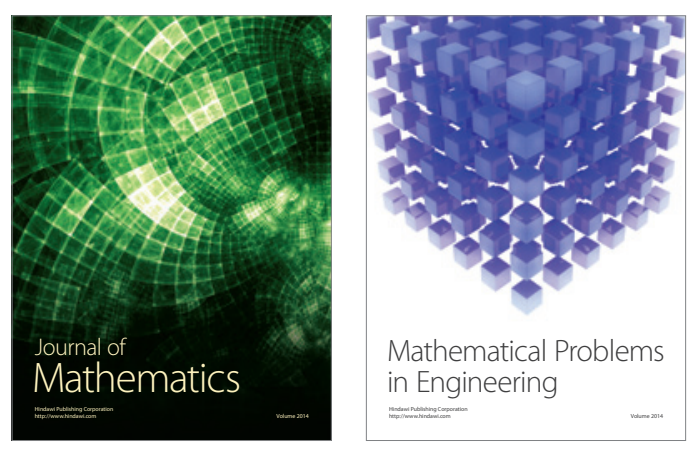

Mathematical Problems in Engineering
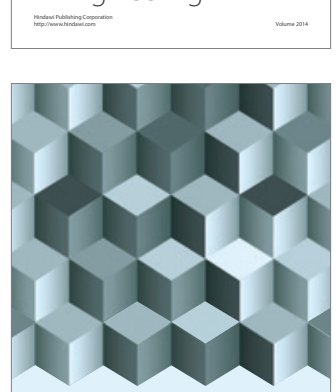

Journal of

Function Spaces
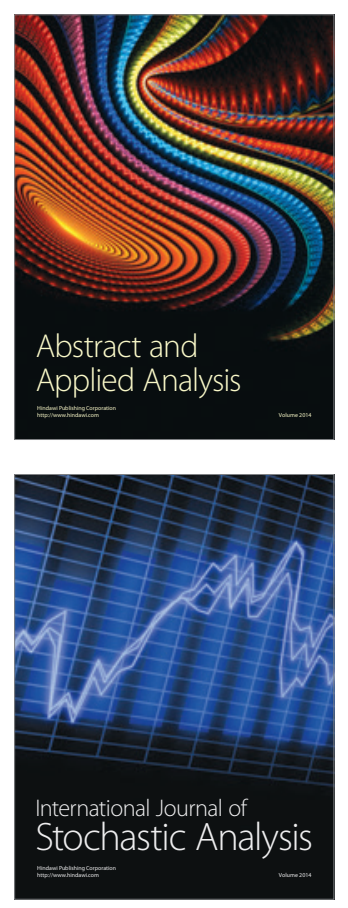

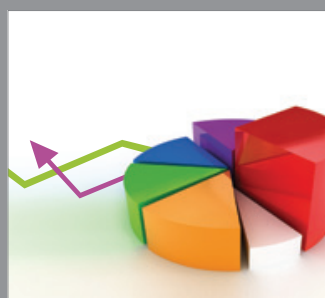

ournal of

Probability and Statistics

Promensencen
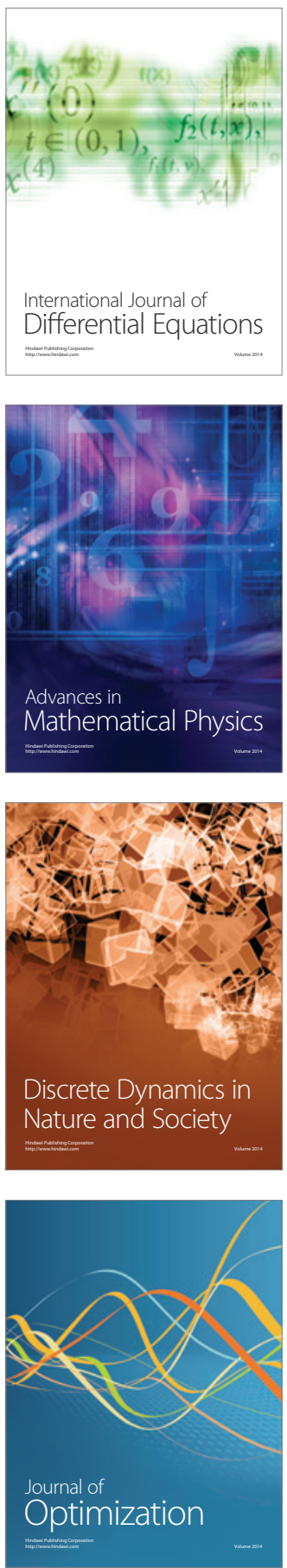